\title{
Hydrodynamic approximation for the nonlinear response of a metal surface
}

\author{
A. Bergara \\ Materia Kondentsatuaren Fisika Saila, Zientzi Fakultatea, Euskal Herriko Unibertsitatea, 644 Posta Kutxatila, \\ 48080 Bilbo, Basque Country, Spain \\ J. M. Pitarke \\ Materia Kondentsatuaren Fisika Saila, Zientzi Fakultatea, Euskal Herriko Unibertsitatea, 644 Posta Kutxatila, \\ 48080 Bilbo, Basque Country, Spain \\ and Donostia International Physics Center (DIPC) and Centro Mixto CSIC-UPV/EHU, Donostia, Basque Country, Spain \\ R. H. Ritchie \\ Oak Ridge National Laboratory, P.O. Box 2008, Oak Ridge, Tennessee 37831-6123 \\ (Received 31 March 1999; revised manuscript received 8 July 1999)
}

\begin{abstract}
We present semiclassical and quantized hydrodynamic models to obtain the quadratic electronic response of a plane-bounded electron gas. Explicit expressions for the dynamic image potential experienced by charged particles moving near a jellium surface are derived, up to third order in the projectile charge. These expressions are employed to compute the image potential at all distances outside the surface. Though nonlinear corrections are found to be more important far inside the solid than outside, our results indicate that the nonlinear image potential is enhanced with respect to the linear image potential by a factor that is for $\mathrm{Al}$ as large as $\sim 1.15$ near the surface in the case of a stationary particle $(\mathrm{V} \rightarrow 0)$ with positive unit charge $e$. [S0163-1829(99)08847-5]
\end{abstract}

\section{INTRODUCTION}

The electronic response of a metal surface to an external perturbation encounters a great variety of important problems in surface science. ${ }^{1}$ Surface spectroscopies employing electrons, photons, atoms, or ions all involve some kind of electronic excitation at the boundary of the surface. In particular, the interaction of charged particles with solids has represented an active field of basic and applied physics, ${ }^{2}$ and recently a great amount of research has been focused on the case of slow ( $\mathrm{v}<0.5 \mathrm{v}_{0}, \mathrm{v}_{0}$ being the Bohr velocity) highly charged ions $\left(Z_{1} \gg 1, Z_{1} e\right.$ being the ion charge) moving near a solid surface. ${ }^{3-7}$ For these projectiles, the parameter $Z_{1} \mathrm{v}_{0} / \mathrm{v}$ is not small and first-order perturbation or, equivalently, linear-response theories are not, a priori, applicable. ${ }^{8}$ In the case of charged particles moving inside a solid, nonlinear effects have been found to be crucial in the interpretation of energy-loss measurements. ${ }^{9-12}$ Nevertheless, the electronic response of metal surfaces to the presence of external charged particles, which differs significantly from that in purely two- or three-dimensional systems, had been described so far within linear-response theory. ${ }^{13}$

A central quantity in the interpretation of ion-surface collisions is the so-called image potential, which represents the interaction between the incoming charge and the polarization charge that it induces on the surface. In the case of a particle of charge $Z_{1} e$ located at a distance $z_{0}$ far from the surface, into the vacuum, this potential approaches the long-range classical Coulomb image potential $V^{\mathrm{im}}=-Z_{1}^{2} e^{2} / 4 z_{0} \cdot{ }^{14}$ For smaller values of $z_{0}$ the image potential differs significantly from its classical limit, the deviation from the classical result increasing as $z_{0}$ decreases. ${ }^{15}$

The classical image potential acting between a point classical charge and a metal surface may be regarded as origi- nating in the coupling with the surface plasmon field. ${ }^{16,17}$ Dynamical corrections to the classical image potential have been discussed in the framework of linear-response theory, ${ }^{18-20}$ and recoil effects have been treated by including the exchange of virtual excitations between the external charge and the medium. ${ }^{21}$ Preliminary results for nonlinear corrections to the image potential associated with the quadratic response of solid surfaces have been reported only very recently. ${ }^{22}$

Theoretical approaches commonly used to describe the electronic response of jellium surfaces can be classified as being either hydrodynamic ${ }^{23}$ in nature or based on the socalled random-phase approximation (RPA). ${ }^{24}$ Hydrodynamic approaches are appealing because of their relative mathematical and computational simplicity, and have been used with great success in the description of collective phenomena at metal surfaces. ${ }^{25}$ Within a hydrodynamic model, one assumes that the collective motion of the electron gas may be described in terms of the displacement of the electrons from their original uniform state, and the electron system is characterized by the electron density and a velocity field. These quantities are then obtained by solving the well-known nonlinear Bloch hydrodynamic equations. If one does not include quantum properties of the electron system we refer to the hydrodynamic model as classical, and as semiclassical if quantum properties are introduced through the definition of the internal energy density. If one quantizes the hydrodynamic Hamiltonian on the basis of the existing normal modes, ${ }^{26}$ we have the so-called quantized hydrodynamic model which allows us to apply standard methods of manybody perturbation theory.

In a previous work, ${ }^{27}$ we used the quantized hydrodynamic model to describe the quadratic response of a homogeneous electron gas. We derived expressions for the qua- 
dratic polarization induced by a moving charged particle, and demonstrated that they coincide with the plasmon-pole approximation $^{28}$ to the more accurate quadratic RPA polarization. $^{29}$

In this paper, we first develop semiclassical and quantized hydrodynamic models to derive the quadratic electronic response of a plane-bounded electron gas, and we then focus on the evaluation of the nonlinear dynamic image potential experienced by charged particles moving parallel to a jellium surface. In Secs. II and III semiclassical and quantized nonlinear hydrodynamic models are presented, respectively. In Sec. IV numerical calculations of both linear and quadratic contributions to the image potential are reported, as a function of the distance from the surface. In Sec. V our conclusions are presented.

\section{SEMICLASSICAL HYDRODYNAMIC MODEL}

Take an inhomogeneous electron system embedded in a neutralizing ionic background. In the hydrodynamic limit, ${ }^{30}$ the total energy of the system can be expressed as ${ }^{31}$ (we use atomic units throughout, i.e., $\left.\hbar=m_{e}=e=1\right)$ :

$$
\begin{aligned}
H= & \frac{1}{2} \int d \mathbf{r} n_{e}(\mathbf{r}, t)|\boldsymbol{\nabla} \psi(\mathbf{r}, t)|^{2}-\frac{1}{2} \int d \mathbf{r} n(\mathbf{r}, t) V(\mathbf{r}, t) \\
& -\int d \mathbf{r} n(\mathbf{r}, t) V_{\text {ext }}(\mathbf{r}, t)+\int d \mathbf{r} G\left[n_{e}(\mathbf{r}, t)\right],
\end{aligned}
$$

where irrotational flow has been assumed, i.e., $\mathbf{u}(\mathbf{r}, t)$ $=-\nabla \psi(\mathbf{r}, t), \mathbf{u}(\mathbf{r}, t)$ being a velocity field, and retardation effects have been neglected. $n_{e}(\mathbf{r}, t)=n_{0}(\mathbf{r})+n(\mathbf{r}, t)$ is the total electron density, with $n(\mathbf{r}, t)$ representing the deviation from the equilibrium static density $n_{0}(\mathbf{r}) . V(\mathbf{r}, t)$ is the induced electric potential, $V_{\text {ext }}(\mathbf{r}, t)$ represents the external perturbation, and $G\left[n_{e}(\mathbf{r}, t)\right]$ represents the exchange, correlation, and internal kinetic energies of the electron system. We neglect exchange-correlation contributions to $G\left[n_{e}(\mathbf{r}, t)\right]$, which we approximate by the Thomas-Fermi functional ${ }^{30}$

$$
G\left[n_{e}(\mathbf{r}, t)\right]=\frac{3}{10}\left(3 \pi^{2}\right)^{2 / 3}\left[n_{e}(\mathbf{r}, t)\right]^{5 / 3} .
$$

From Eq. (1), the basic hydrodynamic equations can be derived, i.e., the continuity equation,

$$
\dot{n}_{e}=\nabla \cdot\left(n_{e} \nabla \psi\right),
$$

and the Bernouilli equation,

$$
\eta \psi+\dot{\psi}+\mu=\frac{1}{2}|\nabla \psi|^{2}-U+\frac{\delta G\left[n_{e}\right]}{\delta n_{e}},
$$

which conserves both momentum and energy. Here, $\mu$ is a Lagrangian multiplier (a constant), and $\eta$ is a positive number representing the internal friction of the electron gas which would appear as a consequence of the interaction with excitations not included in this description. If the external perturbation is generated by a charge density $n_{\text {ext }}(\mathbf{r}, t)$, then the total electric potential $U(\mathbf{r}, t)=V(\mathbf{r}, t)+V_{\text {ext }}(\mathbf{r}, t)$ is obtained from the Poisson equation

$$
\nabla^{2} U=-4 \pi\left[n_{\mathrm{ext}}-n\right]
$$

These are nonlinear equations, difficult to solve. Within perturbation theory, we expand the induced electron density $n(\mathbf{r}, t)$ and the velocity potential $\psi(\mathbf{r}, t)$ in powers of the external perturbation,

$$
n=n_{1}+n_{2}+\cdots
$$

and

$$
\psi=\psi_{1}+\psi_{2}+\cdots,
$$

respectively, assuming that $n_{0} \gg n_{1} \gg n_{2} \gg \ldots$ and $\psi_{1} \gg \psi_{2}$ $\gg$... Equations (3) and (4) are then expanded in powers of the external perturbation, and partial differential equations for the various orders of $n(\mathbf{r}, t)$ and $\psi(\mathbf{r}, t)$ are derived.

\section{A. Linear approximation}

Up to first order in the external perturbation one finds, after introduction of Eq. (2) into Eq. (4), the linearized hydrodynamic equations

$$
\eta \psi_{1}+\dot{\psi}_{1}=-U_{1}+\frac{\beta^{2}}{n_{0}} n_{1}
$$

and

$$
\dot{n}_{1}=n_{0} \nabla^{2} \psi_{1}
$$

where $U_{1}(\mathbf{r}, t)$ is obtained from

$$
\nabla^{2} U_{1}=-4 \pi\left[n_{\mathrm{ext}}-n_{1}\right],
$$

and where $\beta=\sqrt{1 / 3} q_{F}, q_{F}=\left(3 \pi^{2} n_{0}\right)^{1 / 3}$ being the Fermi momentum. Though this is the value of the hydrodynamic speed $\beta$ predicted with use of the Thomas-Fermi functional $G\left[n_{e}(\mathbf{r}, t)\right]$ of Eq. (2), the value $\beta=\sqrt{3 / 5} q_{F}$ is expected to be more appropriate when high frequencies of the order of the plasma frequency are involved. ${ }^{32,33}$

We consider a classical charged particle moving with velocity $\mathbf{v}$ outside of a semi-infinite metallic medium, along a trajectory that is parallel to the surface, thereby approximately simulating the experimental conditions when the projectile approaches the surface at grazing incidence. Hence, we take the external charge density at $\mathbf{r}=\left(\mathbf{r}_{\|}, z\right)$ to be given by the following expression:

$$
n_{\mathrm{ext}}(\mathbf{r}, t)=Z_{1} \delta\left(\mathbf{r}_{\|}-\mathbf{v} t\right) \delta\left(z-z_{0}\right),
$$

the vacuum occupying the half-space $z>0$, and $z_{0}$ being the distance of the trajectory from the metal. After Fourier analyzing both in time and in $\mathbf{r}_{\|}$, one finds the following linearized hydrodynamic equations with variables $(z ; \mathbf{q}, \omega)$, q being the wave vector parallel to the surface:

$$
(-i \omega+\eta) \psi_{1}=-U_{1}+\frac{\beta^{2}}{n_{0}} n_{1}
$$

and

$$
-i \omega n_{1}=n_{0}\left(\psi_{1}^{\prime \prime}-q^{2} \psi_{1}\right),
$$

where the prime denotes the derivative with respect to $z$, $U_{1}(z ; \mathbf{q}, \omega)$ is obtained from

$$
U_{1}^{\prime \prime}-q^{2} U_{1}=-4 \pi n_{\mathrm{ext}}+4 \pi n_{1},
$$


and

$$
n_{\mathrm{ext}}(z ; \mathbf{q}, \omega)=2 \pi Z_{1} \delta(\omega-\mathbf{q} \cdot \mathbf{v}) \delta\left(z-z_{0}\right) .
$$

Assuming a sharp density profile at the surface, i.e., $n_{0}(z)=n_{0} \Theta(-z)[\Theta(z)$ is the Heaviside step function], the appropriate solutions of Eqs. (12) and (13) are

$$
\begin{aligned}
& n_{1}(z ; \mathbf{q}, \omega)=n_{0} A_{1}\left(\Delta_{\mathbf{q}, \omega}^{2}-q^{2}\right) e^{\Delta_{\mathbf{q}, \omega} z} \Theta(-z), \\
& \psi_{1}(z ; \mathbf{q}, \omega)=-\mathrm{i} \omega\left(A_{1} e^{\Delta_{\mathbf{q}, \omega}}+B_{1} e^{q z}\right) \Theta(-z), \\
& U_{1}^{<}(z ; \mathbf{q}, \omega)=\omega_{\mathrm{p}}^{2} A_{1} e^{\Delta_{\mathbf{q}, \omega}}+\omega(\omega+\mathrm{i} \eta) B_{1} e^{q z},
\end{aligned}
$$

and

$$
U_{1}^{>}(z ; \mathbf{q}, \omega)=C_{1} e^{-q z}+Z_{1} \frac{4 \pi^{2}}{q} \delta(\omega-\mathbf{q} \cdot \mathbf{v}) e^{-q\left|z-z_{0}\right|} .
$$

Here, $U_{1}^{<,>}(z ; \mathbf{q}, \omega)$ is the Fourier-transformed electric potential for $z$ (less than, greater than) zero, $\omega_{\mathrm{p}}=\left(4 \pi n_{0}\right)^{1 / 2}$ is the so-called plasma frequency, and

$$
\Delta_{\mathbf{q}, \omega}=\frac{1}{\beta} \sqrt{\omega_{\mathrm{p}}^{2}+\beta^{2} q^{2}-\omega(\omega+\mathrm{i} \eta)} .
$$

The constants $A_{1}, B_{1}$, and $C_{1}$ are evaluated from the boundary conditions at the surface:

$$
\begin{gathered}
A_{1}=-\frac{q}{\Delta_{\mathbf{q}, \omega}} B_{1}, \\
B_{1}=\frac{8 \pi^{2} Z_{1}}{q} \frac{\delta(\omega-\mathbf{q} \cdot \mathbf{v}) e^{-q z_{0}}}{\left[2 \omega(\omega+\mathrm{i} \eta)-\omega_{\mathrm{p}}^{2}\left(1+q / \Delta_{\mathbf{q}, \omega}\right)\right]},
\end{gathered}
$$

and

$$
C_{1}=\frac{4 \pi^{2} Z_{1}}{q} \frac{\omega_{\mathrm{p}}^{2}\left(1-q / \Delta_{\mathbf{q}, \omega}\right) \delta(\omega-\mathbf{q} \cdot \mathbf{v}) e^{-q z_{0}}}{\left[2 \omega(\omega+\mathrm{i} \eta)-\omega_{\mathrm{p}}^{2}\left(1+q / \Delta_{\mathbf{q}, \omega}\right)\right]} .
$$

The induced electric potential $V_{1}(\mathbf{r}, t)$ is the difference between the total and external potentials $U_{1}(\mathbf{r}, t)$ and $V_{\text {ext }}(\mathbf{r}, t)$, respectively. The image potential is defined as half of the induced potential at the position of the projectile times the projectile charge, and one finds

$$
\begin{aligned}
V_{1}^{\mathrm{im}}= & Z_{1}^{2} \frac{\omega_{\mathrm{p}}^{2}}{4 \pi} \int d^{2} \mathbf{q} e^{-2 q z_{0}} \\
& \times \frac{1 / q-1 / \Delta_{\mathbf{q}, \mathbf{q} \cdot \mathbf{v}}}{\left[2 \mathbf{q} \cdot \mathbf{v}(\mathbf{q} \cdot \mathbf{v}+\mathrm{i} \eta)-\omega_{\mathrm{p}}^{2}\left(1+q / \Delta_{\mathbf{q}, \mathbf{q} \cdot \mathbf{v}}\right)\right]} .
\end{aligned}
$$

This agrees with the result obtained using either the specular reflexion $^{34}$ or the semi-classical infinite barrier ${ }^{35}$ model of the surface, as long as the hydrodynamic dielectric response function of the bulk material is used in these models. In particular, for a stationary charged particle $(\mathbf{v}=0)$, one finds

$$
V_{1}^{\mathrm{im}}=\frac{1}{2} Z_{1}^{2} \int d q \frac{q-\Delta_{\mathbf{q}, 0}}{q+\Delta_{\mathbf{q}, 0}} e^{-2 q z_{0}} .
$$

This agrees with the early result of Eguiluz. ${ }^{36}$
Within the hydrodynamic model, one can define two characteristic screening lengths $\lambda$. For a stationary charged particle $(\mathbf{v}=0), \lambda=\beta / \omega_{\mathrm{p}}$, and in the case of a swift charged particle $\left(\mathrm{v} \gg q_{F}\right), \lambda=\mathrm{v} / \omega_{\mathrm{p}}$. In the limit $z_{0} \rightarrow \infty$, the distance $z_{0}$ of the projectile from the surface being much larger than the screening length, one finds the classical image potential

$$
V_{1}^{\mathrm{im}}=-\frac{Z_{1}^{2}}{4 z_{0}}
$$

\section{B. Quadratic approximation}

Up to $N$ th order $(N \geqslant 2)$ in the external perturbation, one finds the hydrodynamic equations:

$$
\begin{aligned}
\eta \psi_{\mathrm{N}}+ & \dot{\psi}_{\mathrm{N}} \\
= & \frac{1}{2} \sum_{\mathrm{m}=1}^{\mathrm{N}-1}\left(\boldsymbol{\nabla} \psi_{\mathrm{N}-\mathrm{m}} \cdot \boldsymbol{\nabla} \psi_{\mathrm{m}}\right)-V_{\mathrm{N}}+\frac{3}{2} \beta^{2} n_{0}^{-2 / 3} \\
& \times \sum_{\mathrm{k}=1}^{\mathrm{N}}\left[\begin{array}{l}
\left.\frac{\prod_{\mathrm{s}=1}^{\mathrm{k}}(5-3 s)}{n_{0}^{\mathrm{k}-2 / 3} 3^{\mathbf{k}}} \sum_{\substack{\mathrm{k}_{1}, \mathrm{k}_{2}, \ldots, \mathrm{k}_{\mathrm{N}}=0 \\
\mathrm{~N}}}^{\mathrm{N}}\left(\prod_{\mathrm{j}=1}^{\mathrm{N}} \frac{n_{\mathrm{j}}^{\mathbf{k}_{j}}}{\mathrm{k}_{\mathrm{j}} !}\right)\right] \\
\sum_{\mathrm{i}=1} \mathrm{k}_{\mathrm{i}}=\mathrm{k} ; \sum_{\mathrm{i}=1} \mathrm{ik}_{\mathrm{i}=\mathrm{N}}
\end{array}\right.
\end{aligned}
$$

and

$$
\dot{n}_{\mathrm{N}}=n_{0} \nabla^{2} \psi_{\mathrm{N}}+\sum_{\mathrm{m}=1}^{\mathrm{N}-1}\left[n_{\mathrm{N}-\mathrm{m}} \nabla^{2} \psi_{\mathrm{m}}+\nabla n_{\mathrm{N}-\mathrm{m}} \cdot \nabla \psi_{\mathrm{m}}\right] \text {, }
$$

where $V_{\mathrm{N}}(\mathbf{r}, t)$ is obtained from

$$
\nabla^{2} V_{\mathrm{N}}=4 \pi n_{\mathrm{N}}
$$

After Fourier transforming in time and in $\mathbf{r}_{\|}$, we obtain for $N=2$ the quadratic approximation

$$
-\mathrm{i}(\omega+\mathrm{i} \eta) \psi_{2}=-V_{2}+\frac{1}{2}\left|\nabla \psi_{1}\right|_{(z ; \mathbf{q}, \omega)}^{2}+\frac{\beta^{2}}{n_{0}} n_{2}-\frac{\beta^{2}}{6 n_{0}^{2}} n_{1}^{2}
$$

and

$$
-\mathrm{i} \omega n_{2}=n_{0}\left(\psi_{2}^{\prime \prime}-q^{2} \psi_{2}\right)+\left[n_{1} \nabla^{2} \psi_{1}+\nabla n_{1} \cdot \nabla \psi_{1}\right]_{(z ; \mathbf{q}, \omega)},
$$

where the prime denotes the derivative with respect to $z$, and the Fourier-transformed potential $V_{2}(z ; \mathbf{q}, \omega)$ is obtained from

$$
V_{2}^{\prime \prime}-q^{2} V_{2}=4 \pi n_{2}
$$

In particular, for a stationary $(\mathbf{v}=0)$ charged particle Eqs. (30) and (31) yield

$$
V_{2}=\frac{\beta^{2}}{n_{0}} n_{2}-\frac{\beta^{2}}{6 n_{0}^{2}} n_{1}^{2}
$$

where $V_{2}(z ; \mathbf{q}, \omega)$ is still obtained from Eq. (32). Solving these equations with the assumption of a sharp density pro- 
file at the surface, quadratic contributions to the induced density and potential are found to be given by the following expressions:

$$
\begin{aligned}
n_{2}(z ; \mathbf{q}, \omega)= & \Theta(-z)\left[A_{2} e^{\Delta_{\mathbf{q}, 0} z}+Z_{1}^{2} \frac{1}{6 n_{0}} \int \frac{d^{2} \mathbf{k}}{(2 \pi)^{2}}\right. \\
& \left.\times e^{\left(\Delta_{\mathbf{k}, 0}+\Delta_{\mathbf{k}-\mathbf{q}, 0}\right) z} \frac{\left(\Delta_{\mathbf{k}, 0}+\Delta_{\mathbf{k}-\mathbf{q}, 0}\right)^{2}-q^{2}}{\left(\Delta_{\mathbf{k}, 0}+\Delta_{\mathbf{k}-\mathbf{q}, 0}\right)^{2}-\Delta_{\mathbf{q}, 0}^{2}} f_{\mathbf{k}} f_{\mathbf{k}-\mathbf{q}}\right],
\end{aligned}
$$

$$
\begin{aligned}
V_{2}^{<}(z ; \mathbf{q}, \omega)= & \frac{4 \pi}{\Delta_{\mathbf{q}, 0}^{2}-q^{2}} A_{2} e^{\Delta_{\mathbf{q}, \omega} z}+B_{2} e^{q z}+Z_{1}^{2} \frac{4 \pi}{6 n_{0}} \\
& \times \int \frac{d^{2} \mathbf{k}}{(2 \pi)^{2}} \frac{f_{\mathbf{k}} f_{\mathbf{k}-\mathbf{q}} e^{\left(\Delta_{\mathbf{k}, 0}+\Delta_{\mathbf{k}-\mathbf{q}, 0}\right) z}}{\left(\Delta_{\mathbf{k}, 0}+\Delta_{\mathbf{k}-\mathbf{q}, 0}\right)^{2}-\Delta_{\mathbf{q}, 0}^{2}},
\end{aligned}
$$

and

$$
V_{2}^{>}(z ; \mathbf{q}, \omega)=C_{2} e^{q z}
$$

where

$$
f_{\mathbf{q}}=\left(\Delta_{\mathbf{q}, 0}-q\right) e^{-q z_{0}},
$$

and $\Delta_{\mathbf{q}, \omega}$ is obtained from Eq. (20). The constants $A_{2}, B_{2}$, and $C_{2}$ are evaluated from the boundary conditions at the surface.

Hence, we find the quadratic contribution to the image potential of a stationary $(\mathbf{v}=0)$ charged particle to be given by the following expression:

$$
\begin{aligned}
V_{2}^{\mathrm{im}}= & -Z_{1}^{3} \frac{\beta^{2}}{6 \pi \omega_{\mathrm{p}}^{4}} \int_{0}^{\infty} d q q \int_{0}^{\infty} d q_{1} q_{1} \\
& \times \int_{0}^{2 \pi} d \theta \frac{f_{\mathbf{q}} f_{\mathbf{q}_{1}} f_{\mathbf{q}-\mathbf{q}_{1}}}{\left(\Delta_{\mathbf{q}, 0}+\Delta_{\mathbf{q}_{1}, 0}+\Delta_{\mathbf{q}-\mathbf{q}_{1}, 0}\right)},
\end{aligned}
$$

$\theta$ being the angle between $\mathbf{q}$ and $\mathbf{q}_{1}$. In the case of a nondispersive electron gas $(\beta=0)$, this contribution to the image potential vanishes. On the other hand, in the limit as $z_{0}$ $\rightarrow \infty$ only the low-momentum form of the integrand of Eq. (38) contributes to the integration, and we find in this limit

$$
\begin{aligned}
V_{2}^{\mathrm{im}}= & -Z_{1}^{3} \frac{1}{18 \pi \omega_{\mathrm{p}}^{2}} \int_{0}^{\infty} d q q \int_{0}^{\infty} d q_{1} q_{1} e^{-\left(q+q_{1}\right) z_{0}} \\
& \times \int_{0}^{2 \pi} d \theta e^{-\left|\mathbf{q}^{-} \mathbf{q}_{1}\right| z_{0}} .
\end{aligned}
$$

Numerical integration yields

$$
V_{2}^{\mathrm{im}}=-Z_{1}^{3} \frac{0.82}{18 \pi \omega_{\mathrm{p}}^{2} z_{0}^{4}},
$$

and, therefore [see Eq. (26)],

$$
V^{\mathrm{im}}=-\frac{Z_{1}^{2}}{4 z_{0}}\left[1+Z_{1} \frac{1.93 \times 10^{-2}}{\left(z_{0} / r_{s}\right)^{3}}+O\left(Z_{1}^{2}\right)\right] .
$$

This is a reasonable approximation for the image potential of a stationary charge, as long as the distance from the surface $z_{0}$ is larger than the characteristic screening length $\beta / \omega_{\mathrm{p}}$, i.e., for $z_{0} \gtrsim \sqrt{r_{s} a_{0}}, a_{0}=\hbar^{2} /\left(m_{e} e^{2}\right)$ being the Bohr radius. We note that in the high-density limit $\left(r_{s} \rightarrow 0\right)$ there is no quadratic contribution to the image potential of a stationary charge, while at metallic densities $\left(r_{s} \sim 2-6\right)$ quadratic corrections might give rise to an image potential that is at $z_{0}$ $\sim \sqrt{r_{s} a_{0}}$ larger than the linear image potential by a factor as large as $1.05-1.3$ in the case of a stationary particle with positive unit charge $e\left(Z_{1}=1\right)$.

\section{QUANTIZED HYDRODYNAMIC MODEL}

Within a quantized hydrodynamic model of the electron gas, we first expand the Hamiltonian of Eq. (1) in powers of the induced electron density $n(\mathbf{r}, t)$. After introduction of the Thomas-Fermi functional of Eq. (2) into Eq. (1), up to third order one finds

$$
H=H_{G}+H_{0}+H_{1}+H_{\text {ext }} \text {, }
$$

where

$$
\begin{gathered}
H_{G}=\frac{3}{10}\left(3 \pi^{2}\right)^{2 / 3} n_{0}^{5 / 3}, \\
H_{0}=\int d \mathbf{r}\left[\frac{1}{2} n_{0}|\nabla \psi|^{2}+\frac{\beta^{2}}{2 n_{0}} n^{2}-\frac{1}{2} n V\right], \\
H_{1}=\int d \mathbf{r}\left[\frac{1}{2} n|\nabla \psi|^{2}-\frac{\beta^{2}}{18 n_{0}^{2}} n^{3}\right],
\end{gathered}
$$

and

$$
H_{\mathrm{ext}}=-\int d \mathbf{r} n V_{\mathrm{ext}} .
$$

$H_{G}$ is the Thomas-Fermi ground state of the static unperturbed electron system, $H_{0}$ represents the linear deviation from the ground state, $H_{1}$ appears as a consequence of the nonlinearity of the electron system, and $H_{\text {ext }}$ represents the contribution to the Hamiltonian from the coupling with the external charged particle.

We consider, as in the previous section, a semi-infinite electron system embedded in a neutralizing ionic background, assuming a sharp electron-density profile at the surface. For each value of $\mathbf{q}$ (the wave vector parallel to the surface) there exist both bulk and surface normal modes of oscillation with frequencies given by the following dispersion relations:

$$
\left(\omega_{\mathbf{q}, p}^{B}\right)^{2}=\omega_{\mathrm{p}}^{2}+\beta^{2}\left(q^{2}+p^{2}\right)
$$

and

$$
\left(\omega_{\mathbf{q}}^{S}\right)^{2}=\frac{1}{2}\left[\omega_{\mathrm{p}}^{2}+\beta^{2} q^{2}+\beta q\left(2 \omega_{\mathrm{p}}^{2}+\beta^{2} q^{2}\right)^{1 / 2}\right]
$$

respectively, where $\beta$ represents the speed of propagation of hydrodynamic disturbances in the electron system. As in the previous section, we choose $\beta=\sqrt{3 / 5} q_{F}$. 
Now we follow Ref. 26 to quantize the Hamiltonian of Eq. (42) on the basis of the normal modes corresponding to Eqs. (47) and (48), which we shall refer to after quantization as bulk and surface plasmons, respectively. We find

$$
H_{0}=H_{0}^{S}+H_{0}^{B},
$$

$H_{0}^{B}$ and $H_{0}^{S}$ being free bulk and surface plasmon Hamiltonians, respectively:

$$
H_{0}^{B}=\frac{1}{\Omega} \sum_{\mathbf{q}, p>0}\left[1 / 2+\omega_{\mathbf{q}, p}^{B}\right] a_{\mathbf{q}, p}^{\dagger}(t) a_{\mathbf{q}, p}(t)
$$

and

$$
H_{0}^{S}=\frac{1}{A} \sum_{\mathbf{q}}\left[1 / 2+\omega_{\mathbf{q}}^{S}\right] b_{\mathbf{q}}^{\dagger}(t) b_{\mathbf{q}}(t) .
$$

Here $\Omega$ and $A$ represent the normalization volume and the normalization area of the surface, respectively, and $a_{\mathbf{q}, p}(t)$ and $b_{\mathbf{q}}(t)$ are Bose-Einstein operators that annihilate bulk and surface plasmons with wave vectors $(\mathbf{q}, p)$ and $\mathbf{q}$, respectively. The quantized $H_{1}$ hamiltonian, which contains the quadratic electronic response of the electron system, will be consider below. For the Hamiltonian containing the coupling between the external particle and either bulk or surface plasmon fields, one finds

$$
H_{\mathrm{ext}}=H_{\mathrm{ext}}^{S}+H_{\mathrm{ext}}^{B},
$$

where

$$
H_{\mathrm{ext}}^{B / S}=\int d \mathbf{r} \rho_{\mathrm{ext}}(\mathbf{r}, t) \phi^{B / S}(\mathbf{r}, t),
$$

$\phi^{B / S}(\mathbf{r}, t)$ representing operators corresponding to the scalar electric potential due to bulk/surface plasmons. Outside the metal $(z>0)$

$$
\phi^{B}(\mathbf{r}, t)=-\frac{1}{\Omega} \sum_{\mathbf{q}, p>0} f_{\mathbf{q}, p}^{B}(z) e^{\mathbf{i q} \cdot \mathbf{r}_{\|}} \chi_{\mathbf{q}, p}^{B}(t)
$$

and

$$
\phi^{S}(\mathbf{r}, t)=-\frac{1}{A} \sum_{\mathbf{q}} f_{\mathbf{q}}^{S}(z) e^{\mathbf{i q} \cdot \mathbf{r}_{\|}} \chi_{\mathbf{q}}^{S}(t),
$$

$\chi_{\mathbf{q}, p}^{B}(t)$ and $\chi_{\mathbf{q}}^{S}(t)$ representing operators associated to the electron density induced by the excitation of bulk and surface plasmon fields, respectively,

$$
\chi_{\mathbf{q}, p}^{B}(t)=a_{\mathbf{q}, p}^{\dagger}(t)+a_{-\mathbf{q}, p}(t)
$$

and

$$
\chi_{\mathbf{q}}^{S}(t)=b_{\mathbf{q}}^{\dagger}(t)+b_{-\mathbf{q}}(t),
$$

and $f_{\mathbf{q}, p}^{B}(z)$ and $f_{\mathbf{q}}^{S}(z)$ being bulk and surface coupling functions,

$$
f_{\mathbf{q}, p}^{B}(z)=\frac{\sqrt{2 \pi / \omega_{\mathbf{q}, p}^{B}} \omega_{\mathrm{p}} p e^{-q z}}{\left[p^{4}+p^{2}\left(q^{2}+\omega_{\mathrm{p}}^{2} / \beta^{2}\right)+\omega_{\mathrm{p}}^{4} /\left(4 \beta^{4}\right)\right]^{1 / 2}}
$$

and

$$
f_{\mathbf{q}}^{S}(z)=\frac{\sqrt{\pi \gamma_{\mathbf{q}} / \omega_{\mathbf{q}}^{S}} \omega_{\mathrm{p}}}{\left[q\left(q+2 \gamma_{\mathbf{q}}\right)\right]^{1 / 2}} e^{-q z} .
$$

Here $\gamma_{\mathbf{q}}$ represents the so-called inverse decay length of surface plasmon charge fluctuations, ${ }^{36}$

$$
\gamma_{\mathbf{q}}=\frac{1}{2 \beta}\left[-\beta q+\sqrt{2 \omega_{\mathbf{p}}^{2}+\beta^{2} q^{2}}\right] .
$$

In the absence of electron-gas dispersion $(\beta=0)$, the scalar electric potential $\phi^{B}(\mathbf{r}, t)$ due to bulk plasmons vanishes outside the surface; hence, in this case probes exterior to the solid can only generate surface excitations.

We derive the potential induced by the presence of the external perturbing charge as the expectation value of the total scalar potential operator, ${ }^{37}$

$$
V(\mathbf{r}, t)=\frac{\left\langle\Psi_{0}\left|\phi_{H}^{B}+\phi_{H}^{S}\right| \Psi_{0}\right\rangle}{\left\langle\Psi_{0} \mid \Psi_{0}\right\rangle}
$$

where $\left|\Psi_{0}\right\rangle$ is the Heisenberg ground state of the interacting system, and where $\phi_{H}^{B}(\mathbf{r}, t)$ and $\phi_{H}^{S}(\mathbf{r}, t)$ are the operators of Eqs. (54) and (55) in the Heisenberg picture. Equation (61) can be rewritten as follows

$$
V(\mathbf{r}, t)=\frac{\left\langle\Phi_{0}\left|U^{\dagger}(t,-\infty)\left[\phi_{I}^{B}+\phi_{I}^{S}\right] U(t,-\infty)\right| \Phi_{0}\right\rangle}{\left\langle\Phi_{0}\left|U^{\dagger}(t,-\infty) U(t,-\infty)\right| \Phi_{0}\right\rangle}
$$

where $\left|\Phi_{0}\right\rangle$ represents the ground state of an interacting electron system described by the free plasmon Hamiltonian $H_{0}$, and $U\left(t_{1}, t_{0}\right)$ is the evolution operator,

$$
U\left(t_{1}, t_{0}\right)=T\left\{\exp \left[-\mathrm{i} \int_{t_{0}}^{t_{1}} d t\left(H_{1}^{I}+H_{\text {ext }}^{I}\right)\right]\right\},
$$

$T$ being the chronological operator, and $H_{1}^{I} / H_{\mathrm{ext}}^{I}$ representing the Hamiltonians $H_{1} / H_{\text {ext }}$ in the interaction picture.

\section{A. Linear approximation}

Up to first order in the external perturbation one finds, after introduction of Eq. (63) into Eq. (62), the linear contribution to the induced potential:

$$
V_{1}(\mathbf{r}, t)=V_{1}^{B}+V_{1}^{S},
$$

where

$$
\begin{aligned}
V_{1}^{B}(\mathbf{r}, t)= & Z_{1} \int \frac{d^{2} \mathbf{q}}{(2 \pi)^{2}} \int_{0}^{\infty} \frac{d p}{2 \pi} \\
& \times f_{\mathbf{q}, p}^{B}(z) f_{\mathbf{q}, p}^{B}\left(z_{0}\right) D_{\mathbf{q}, p}^{B}(\mathbf{q} \cdot \mathbf{v}) e^{\mathbf{i q} \cdot\left(\mathbf{r}_{\|}-\mathbf{v} t\right)}
\end{aligned}
$$

and

$$
V_{1}^{S}(\mathbf{r}, t)=Z_{1} \int \frac{d^{2} \mathbf{q}}{(2 \pi)^{2}} f_{\mathbf{q}}^{S}(z) f_{\mathbf{q}}^{S}\left(z_{0}\right) D_{\mathbf{q}}^{S}(\mathbf{q} \cdot \mathbf{v}) e^{\mathbf{i q} \cdot\left(\mathbf{r}_{\|}-\mathbf{v}, t\right)},
$$

$D_{\mathbf{q}, p}^{B}(\omega)$ and $D_{\mathbf{q}}^{S}(\omega)$ representing retarded Green's functions for the operators $\chi_{\mathbf{q}, p}^{B}(t)$ and $\chi_{\mathbf{q}}^{S}(t)$, respectively: 


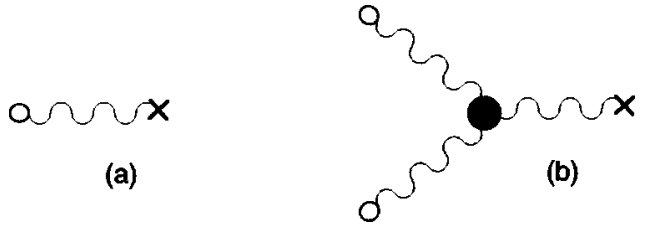

FIG. 1. Feynman diagrams representing first- (a) and second(b) order contributions to the electric potential induced by an external charged particle. The external perturbation is represented by white points, and the cross represents a test positive unit charge. Wavy lines represent plasmon propagators and the black point, joining three plasmon lines, describes the nonlinear interaction between three excitations

$$
D_{\mathbf{q}, p}^{B}(\omega)=\frac{2 \omega_{\mathbf{q}, p}^{B}}{\omega(\omega+\mathrm{i} \eta)-\left(\omega_{\mathbf{q}, p}^{B}\right)^{2}}
$$

and

$$
D_{\mathbf{q}}^{S}(\omega)=\frac{2 \omega_{\mathbf{q}}^{S}}{\omega(\omega+\mathrm{i} \eta)-\left(\omega_{\mathbf{q}}^{S}\right)^{2}}
$$

Equation (64) agrees with the linear contribution to the induced potential obtained, within the semiclassical hydrodynamic model, as the difference between the Fourier transform of the total potential of Eq. (19) and the external potential $V_{\text {ext }}(\mathbf{r}, t)$. Within the semiclassical approach, the role played by bulk and surface plasmons goes unnoticed; however, the quantized hydrodynamic model provides explicit separate expressions for the contributions to the induced potential coming from the coupling with bulk and surface plasmons. The role that bulk and surface plasmon excitation plays on the energy loss of charged particles interacting with metal surfaces has been investigated recently, ${ }^{38}$ showing that bulk plasmons are excited even in the case of charged particles that do not penetrate into the solid.

\section{B. Quadratic approximation}

Quantizing the Hamiltonian $H_{1}$ of Eq. (45) on the basis of both bulk and surface plasmons is a difficult task, because of the interaction between bulk and surface plasmon fields. Hence, for the description of the nonlinear response to a charged particle moving outside of a semi-infinite medium, now we neglect bulk-plasmon contributions and find the following expression for the quantized $H_{1}$ Hamiltonian:

$$
\begin{aligned}
H_{1}= & \frac{1}{A^{2}} \sum_{\mathbf{q}_{1}} \sum_{\mathbf{q}_{2}}\left[-\Lambda_{\mathbf{q}_{1}, \mathbf{q}_{2}} \dot{\chi}_{\mathbf{q}_{1}}^{S}(t) \dot{\chi}_{\mathbf{q}_{2}}^{S}(t)\right. \\
& \left.+\mathcal{P}_{\mathbf{q}_{1}, \mathbf{q}_{2}} \chi_{\mathbf{q}_{1}}^{S}(t) \chi_{\mathbf{q}_{2}}^{S}(t)\right] \chi_{-\left(\mathbf{q}_{1}+\mathbf{q}_{2}\right)}^{S}(t),
\end{aligned}
$$

where

$$
\begin{aligned}
\Lambda_{\mathbf{q}_{1}, \mathbf{q}_{2}}= & \frac{\gamma_{\mathbf{q}_{1}+\mathbf{q}_{2}}+\left|\mathbf{q}_{1}+\mathbf{q}_{2}\right|}{\left(\gamma_{\mathbf{q}_{1}}-q_{1}\right)\left(\gamma_{\mathbf{q}_{2}}-q_{2}\right) \sqrt{2 n_{0} q_{1} q_{2} \omega_{\mathbf{q}_{1}}^{S} \omega_{\mathbf{q}_{2}}^{S} \omega_{\mathbf{q}_{1}+\mathbf{q}_{2}}^{S}}} \\
& \times\left[\frac{\left|\mathbf{q}_{1}+\mathbf{q}_{2}\right| \gamma_{\mathbf{q}_{1}+\mathbf{q}_{2}} \gamma_{\mathbf{q}_{1}} \gamma_{\mathbf{q}_{2}}}{\left(q_{1}+2 \gamma_{\mathbf{q}_{1}}\right)\left(q_{2}+2 \gamma_{\mathbf{q}_{2}}\right)\left(\left|\mathbf{q}_{1}+\mathbf{q}_{2}\right|+2 \gamma_{\mathbf{q}_{1}+\mathbf{q}_{2}}\right)}\right]\left[\frac{q_{1} q_{2}\left(\mathbf{q}_{1} \cdot \mathbf{q}_{2}-\gamma_{\mathbf{q}_{1}} \gamma_{\mathbf{q}_{2}}\right)}{\gamma_{\mathbf{q}_{1}}+\gamma_{\mathbf{q}_{2}}+\gamma_{\mathbf{q}_{1}+\mathbf{q}_{2}}}-\frac{q_{1} \gamma_{\mathbf{q}_{2}}\left(\mathbf{q}_{1} \cdot \mathbf{q}_{2}-\gamma_{\mathbf{q}_{1}} q_{2}\right)}{\gamma_{\mathbf{q}_{1}}+q_{2}+\gamma_{\mathbf{q}_{1}+\mathbf{q}_{2}}}\right. \\
& \left.+\frac{q_{2} \gamma_{\mathbf{q}_{1}}\left(\mathbf{q}_{1} \cdot \mathbf{q}_{2}-q_{1} \gamma_{\mathbf{q}_{2}}\right)}{q_{1}+\gamma_{\mathbf{q}_{2}}+\gamma_{\mathbf{q}_{1}+\mathbf{q}_{2}}}-\frac{\gamma_{\mathbf{q}_{1}} \gamma_{\mathbf{q}_{2}}\left(\mathbf{q}_{1} \cdot \mathbf{q}_{2}-q_{1} q_{2}\right)}{q_{1}+q_{2}+\gamma_{\mathbf{q}_{1}+\mathbf{q}_{2}}}\right]
\end{aligned}
$$

and

$$
\mathcal{P}_{\mathbf{q}_{1}, \mathbf{q}_{2}}=-\frac{\beta^{2}}{18 n_{0}^{1 / 2}}\left[\frac{q_{1} q_{2}\left|\mathbf{q}_{1}+\mathbf{q}_{2}\right| \gamma_{\mathbf{q}_{1}}}{\omega_{\mathbf{q}_{1}}^{S} \omega_{\mathbf{q}_{2}}^{S} \omega_{\mathbf{q}_{1}+\mathbf{q}_{2}}^{S}\left(q_{1}+2 \gamma_{\mathbf{q}_{1}}\right)} \frac{\gamma_{\mathbf{q}_{2}} \gamma_{\mathbf{q}_{1}+\mathbf{q}_{2}}}{\left(q_{2}+2 \gamma_{\mathbf{q}_{2}}\right)\left(\left|\mathbf{q}_{1}+\mathbf{q}_{2}\right|+2 \gamma_{\mathbf{q}_{1}+\mathbf{q}_{2}}\right)}\right]^{1 / 2} \frac{\left(q_{1}+\gamma_{\mathbf{q}_{1}}\right)\left(q_{2}+\gamma_{\mathbf{q}_{2}}\right)\left(\left|\mathbf{q}_{1}+\mathbf{q}_{2}\right|+\gamma_{\mathbf{q}_{1}+\mathbf{q}_{2}}\right)}{\gamma_{\mathbf{q}_{1}}+\gamma_{\mathbf{q}_{2}}+\gamma_{\mathbf{q}_{1}}+\mathbf{q}_{2}}
$$

The first term in Eq. (69) comes from the kinetic energy of fluid flow, $\int d \mathbf{r} n|\nabla \psi|^{2} / 2$, while the second term, which is proportional to $\beta^{2}$, comes from the internal energy $G[n]$ of Eq. (2).

Introducing Eqs. (52) and (69) into Eq. (63), and Eq. (63) into Eq. (62), the quadratic contribution to the induced potential is found to be given by the following expression:

$$
\begin{aligned}
V_{2}(\mathbf{r}, t)= & -Z_{1}^{2} \int \frac{d^{2} \mathbf{q}}{(2 \pi)^{2}} \frac{d^{2} \mathbf{q}_{1}}{(2 \pi)^{2}} f_{\mathbf{q}}^{S}\left(z+z_{0}\right) f_{\mathbf{q}_{1}}^{S}\left(z_{0}\right) f_{\mathbf{q}^{-} \mathbf{q}_{1}}^{S}\left(z_{0}\right) D_{\mathbf{q}}^{S}(\omega) D_{\mathbf{q}_{1}}^{S}\left(\omega_{1}\right) D_{\mathbf{q}-\mathbf{q}_{1}}^{S}\left(\omega-\omega_{1}\right) \\
& \times\left[\omega \omega_{1} \Lambda_{-\mathbf{q}, \mathbf{q}_{1}}-\omega\left(\omega-\omega_{1}\right) \Lambda_{-\mathbf{q}, \mathbf{q}-\mathbf{q}_{1}}+\omega_{1}\left(\omega-\omega_{1}\right) \Lambda_{\mathbf{q}_{1}, \mathbf{q}-\mathbf{q}_{1}}+3 \mathcal{P}_{\mathbf{q},-\mathbf{q}_{1}}\right] e^{\mathbf{i q} \cdot\left(\mathbf{r}_{\|}-\mathbf{v} t\right)},
\end{aligned}
$$

where $\omega=\mathbf{q} \cdot \mathbf{v}$ and $\omega_{1}=\mathbf{q}_{1} \cdot \mathbf{v}$. Linear and quadratic contributions to the induced potential [see Eqs. (64) and (72)] can be represented diagrammatically as in Fig. 1. As for the quadratic contribution, the external perturbation (white circles) acts twice on the electron gas through plasmon propagators (wavy lines), thereby creating an induced potential at point $\mathbf{r}$ and time $t$ (crosses). 
In particular, for a stationary charged particle we have $\omega=\omega_{1}=0$, thereby only the internal kinetic energy of Eq. (2) contributing to the quadratic induced potential. For the quadratic contribution to the image potential of a stationary charged particle we find

$$
\begin{aligned}
V_{2}^{\mathrm{im}}= & -Z_{1}^{3} \frac{\beta^{2} \omega_{\mathrm{p}}^{2}}{6 \pi} \int_{0}^{\infty} d q q \int_{0}^{\infty} d q_{1} q_{1} e^{-\left(q+q_{1}\right) z_{0}} \int_{0}^{2 \pi} d \theta \\
& \times \frac{\gamma_{\mathbf{q}} \gamma_{\mathbf{q}_{1}} \gamma_{\mathbf{q}-\mathbf{q}_{1}} e^{-\left(\mathbf{q}-\mathbf{q}_{1}\right) z_{0}}}{\left(\omega_{\mathbf{q}}^{S} \omega_{\mathbf{q}_{1}}^{S} \omega_{\mathbf{q}-\mathbf{q}_{1}}^{S}\right)^{2}\left(\gamma_{\mathbf{q}}+\gamma_{\mathbf{q}_{1}}+\gamma_{\mathbf{q}-\mathbf{q}_{1}}\right)} \frac{\left(q+\gamma_{\mathbf{q}}\right)\left(\mathbf{q}_{1}+\gamma_{\mathbf{q}_{1}}\right)\left(\left|\mathbf{q}-\mathbf{q}_{1}\right|+\gamma_{\mathbf{q}-\mathbf{q}_{1}}\right)}{\left(q+2 \gamma_{\mathbf{q}}\right)\left(q_{1}+2 \gamma_{\mathbf{q}_{1}}\right)\left(\left|\mathbf{q}-\mathbf{q}_{1}\right|+2 \gamma_{\mid \mathbf{q}-\mathbf{q}_{1}}\right)},
\end{aligned}
$$

which in the case of a nondispersive electron gas $(\beta=0)$ vanishes. Here $\theta$ is the angle between $\mathbf{q}$ and $\mathbf{q}_{1}$, and in the limit as $z_{0} \rightarrow \infty$ Eq. (73) yields

$$
V_{2}^{\mathrm{im}}=-Z_{1}^{3} \frac{0.41}{18 \pi \omega_{\mathrm{p}}^{2} z_{0}^{4}} .
$$

This quadratic contribution to the image potential is half the result obtained within the semiclassical hydrodynamic model [see Eq. (40)]. In the case of a stationary charged particle the whole quadratic contribution to the image potential comes from the second term in Eq. (45), i.e., from the linearly induced electron density acting twice on the external charge. The total electron density $n_{1}$ induced at the surface by a stationary charged particle is, in the limit $z_{0} \rightarrow \infty, \sqrt{2}$ times the electron density $n_{1}^{S}$ induced through coupling with surface plasmons (see the Appendix). As a consequence, the total quadratic contribution to the image potential is in this limit [see Eq. (40)] twice as large as the quadratic contribution of Eq. (74), which has been deduced by neglecting the coupling with bulk plasmons.

\section{RESULTS}

Figure 2 shows plots of the linear contribution to the image potential of a particle with unit charge $e\left(Z_{1}=1\right)$ traveling parallel to the surface of a semi-infinite electron gas characterized by a static electron density $n_{0}$ equal to the average electron density in the conduction band of aluminum $\left(r_{s}\right.$ 2). ${ }^{39}$ These plots are shown as a function of $z_{0}$, the distance from the surface, and the speed is taken to be $v=0$ [Fig. 2(a)] and $v=2$ [Fig. 2(b)]. Contributions from couplings with bulk and surface plasmon fields, as obtained from Eqs. (65) and (66), respectively, are represented separately by dashed and dotted lines, and the total linear contribution to the image potential, obtained from either Eq. (24) or Eq. (64), is represented by a solid line. For comparison, the classical image potential of Eq. (26) is represented by a dashed-dotted line, showing that it converges with the full linear result when the distance $z_{0}$ is well above the screening length, i.e., $z_{0} \gtrsim \sqrt{r_{s} a_{0}}$ for $\mathrm{v}=0$ and $z_{0} \sim 2 \sqrt{r_{s} a_{0}}$ for $\mathrm{v}=2$.

Quadratic contributions to the image potential of a particle with unit charge $e\left(Z_{1}=1\right)$ traveling, as in Fig. 2, parallel to the surface of a plane-bounded electron gas are depicted in Fig. 3. The electron-density parameter $r_{s}$ and the velocity of the external charged particle are the same as those considered in Fig. 2. In the case of a stationary charged par- ticle [Fig. 3(a)], the contribution from coupling with the surface plasmon field, as obtained from Eq. (73), is represented (dotted line) together with the total quadratic contribution (solid line), obtained from Eq. (38). At large distances from the surface $\left(z_{0} \rightarrow \infty\right)$, the total quadratic contribution to the image potential is twice as large as the quadratic contribution from surface plasmon excitation, as discussed after Eq. (74). At smaller values of $z_{0}$, the quadratic contribution from the bulk channel becomes dominant, the total quadratic image potential being near the surface larger than the quadratic contribution from the surface channel by a factor of $\sim 10$. The
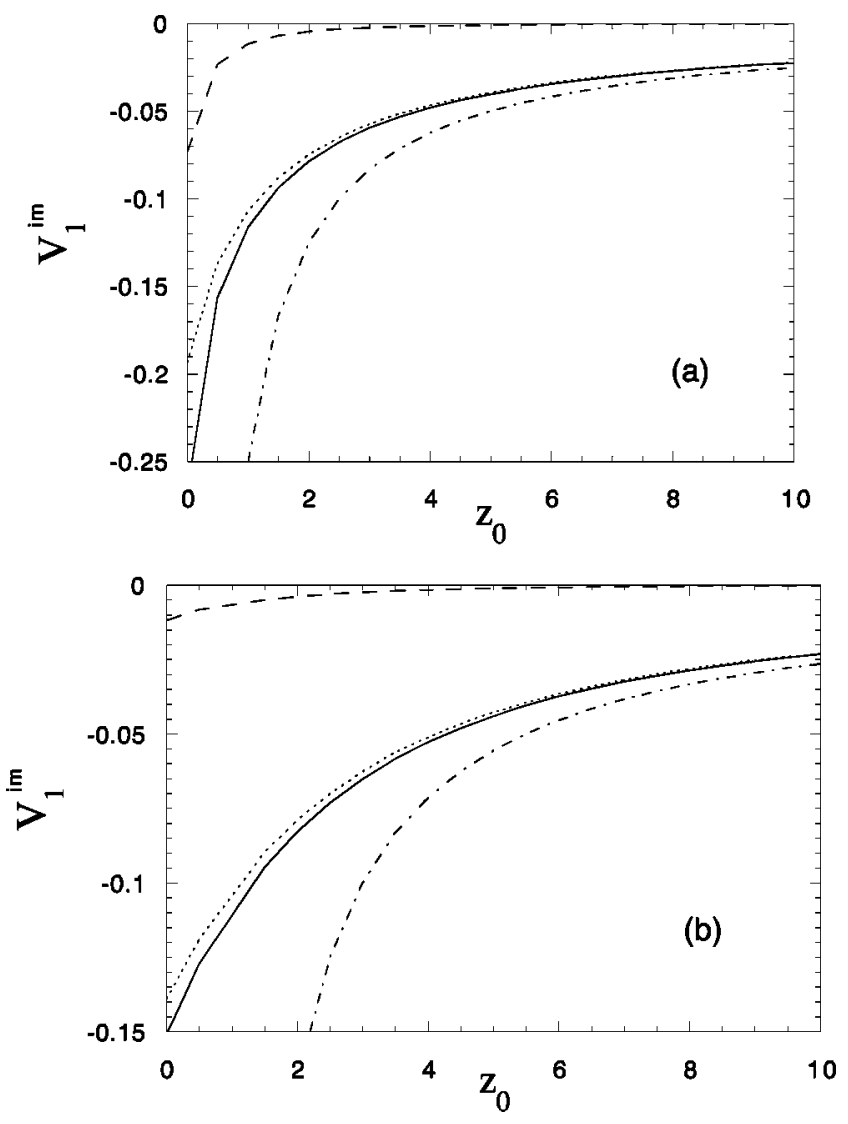

FIG. 2. Linear contribution to the image potential of a particle with charge $Z_{1}=1$ and speed $v=0$ (a) and $v=2$ (b) traveling parallel to the surface of a semi-infinite electron gas with $r_{s}=2$, as a function of the distance $z_{0}$ from the surface. Solid lines represent the full linear contribution to the image potential. Dashed and dotted lines represent contributions from the excitation of bulk and surface plasmons, respectively. The classical image potential of Eq. (26) is represented by a dashed-dotted line. 

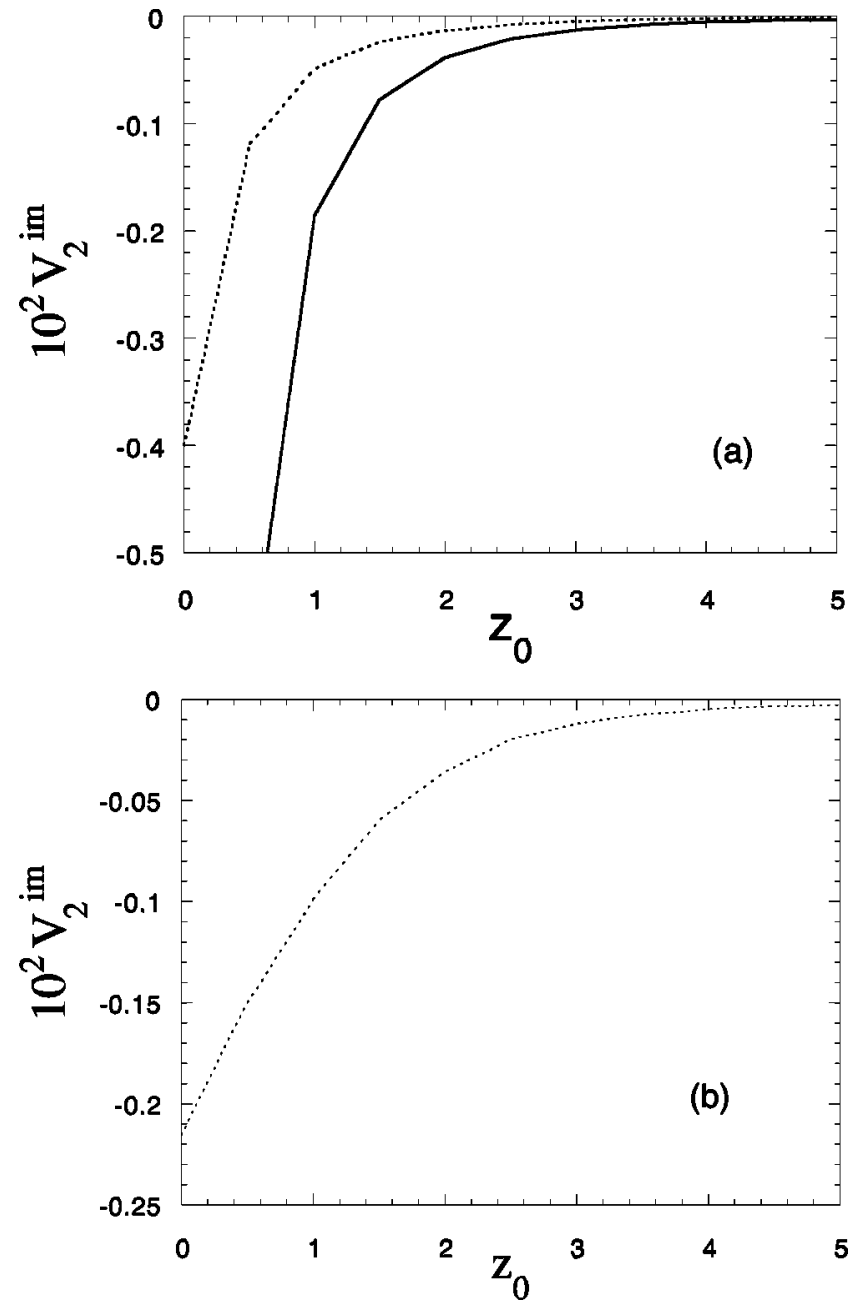

FIG. 3. As in Fig. 2, for the quadratic contribution to the image potential of a particle with charge $Z_{1}=1$. Dotted lines represent the quadratic contribution from to the image potential coming from surface plasmon excitation. In the case of a stationary particle ( $\mathrm{V}$ $=0$ ), the full quadratic contribution to the image potential is represented by a solid line.

quadratic contribution from the surface channel to the image potential of a charged particle moving with speed $v=2$, as obtained from Eq. (72), is represented by a dotted line in Fig. 3(b).

Figure 4 exhibits by a solid line, as a function of the distance $z_{0}$ from the surface, the ratio between full quadratic [solid line of Fig. 3(a)] and linear [solid line of Fig. 2(a)] contributions to the image potential of a stationary particle with unit charge $e\left(Z_{1}=1\right)$. For comparison, the ratio $Z_{1} 1.93 \times 10^{-2}\left(z_{0} / r_{s}\right)^{-3}$, as obtained from Eq. (41), is represented by a dashed-dotted line, showing that it converges with the full ratio (solid line) when the distance $z_{0}$ is well above the screening length, i.e., $z_{0} \gtrsim \sqrt{r_{s} a_{0}}$.

\section{CONCLUSIONS}

First of all, we have presented semiclassical and quantized hydrodynamic models to obtain the quadratic electronic response of a semi-infinite electron gas. Then, we have derived explicit expressions for the dynamic image potential experienced by charged particles traveling parallel to a jel-

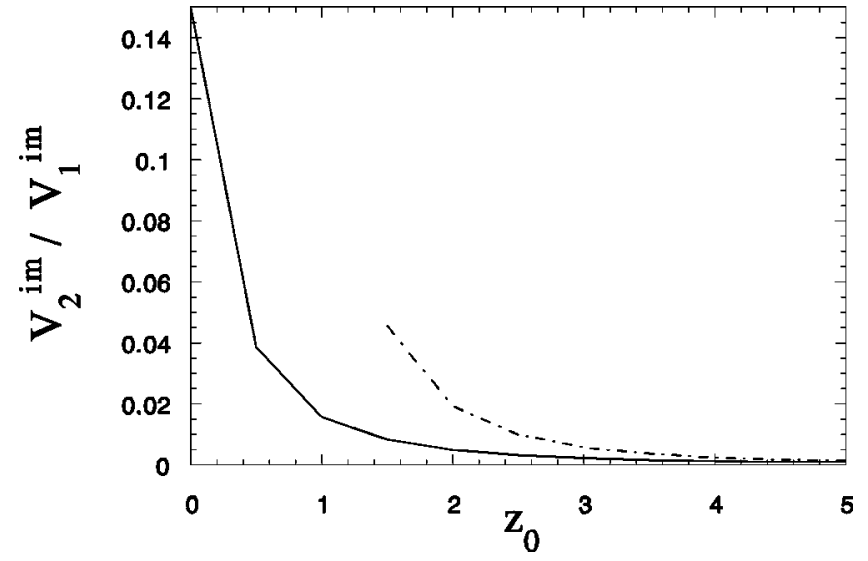

FIG. 4. Ratio between quadratic and linear contributions to the image potential of a stationary particle $(\mathrm{V}=0)$ with charge $Z_{1}=1$ that is located outside the surface of a semi-infinite electron gas with $r_{s}=2$ at a distance $z_{0}$ from the surface. The solid line represents the ratio between full calculations of quadratic and linear image potentials. The dashed-dotted line represents the approximate ratio $Z_{1} 1.93 \times 10^{-2}\left(z_{0} / r_{s}\right)^{-3}$, taken from Eq. (41).

lium surface, up to third order in the projectile charge. In the case of a stationary charged particle the total quadratic contribution to the image potential has been found to be, at large distances from the surface, twice as large as the quadratic contribution coming from the surface plasmon field. Near the surface, the total quadratic contribution to the image potential of a charged particle with $v=0$ has been found to be larger than the quadratic contribution from the surface channel by a factor of $\sim 10$. As the speed of the moving charged particle increases, linear contributions to the image potential coming from the bulk channel have been found to decrease, and quadratic contributions from coupling with the bulk plasmon field are also expected to decrease with increasing velocity.

Though nonlinear corrections are found to be more important far inside the solid ${ }^{29}$ than outside, our results indicate that the nonlinear image potential is enhanced with respect to the linear image potential by a factor that is for aluminum as large as $\sim 1.15$ near the surface in the case of a stationary charged particle $(\mathrm{v}=0)$ with unit charge $e\left(Z_{1}=1\right)$. At large distances $\left(z_{0} \gg \sqrt{r_{s} a_{0}}\right)$ from the surface, the ratio between quadratic and linear contributions to the image potential of a stationary charged particle decreases with the distance $z_{0}$ as $Z_{1} 1.93 \times 10^{-2}\left(z_{0} / r_{s}\right)^{-3}$, showing that it vanishes at high electron densities.

As the speed of the moving charged particle increases, quadratic contributions to the image potential are found to be very small. In particular, in the case of a projectile of charge $Z_{1}=10$ moving with speed $\mathrm{v}=2$ near the metal surface, contributions to the quadratic image potential from coupling with the surface plasmon field have been found to enhance the linear image potential near the surface by a a factor of $\sim 1.14$.

\section{ACKNOWLEDGMENTS}

A.B. and J.M.P. wish to acknowledge partial support by the Basque Unibertsitate eta Ikerketa Saila and the Spanish Ministerio the Educación y Cultura. 


\section{APPENDIX}

Here we use both semiclassical and quantized hydrodynamic models to evaluate the first-order electron density $n_{1}$, which we assume to be induced by a stationary particle ( $\mathbf{v}$ $=0$ ) of charge $Z_{1}$ that is located far from the surface, i.e., $z_{0} \rightarrow \infty$.

Within the semiclassical hydrodynamic model, this quantity is easily found from Eq. (16) to be given by the following expression:

$$
n_{1}(z)=Z_{1} \frac{\omega_{\mathrm{p}}}{2 \pi \beta} \frac{e^{\omega_{\mathrm{p}} z / \beta}}{z_{0}^{2}} \Theta(-z)
$$

Within the quantized hydrodynamic model, the operators corresponding to the induced electron density due to bulk and surface plasmons are obtained as follows:

$$
\hat{n}^{B}(\mathbf{r}, t)=-\frac{1}{\Omega} \sum_{\mathbf{q}, p>0} g_{\mathbf{q}, p}^{B}(z) e^{\mathbf{i q} \cdot \mathbf{r}_{\|}} \chi_{\mathbf{q}, p}^{B}(t) \Theta(-z)
$$

and

$$
\hat{n}^{S}(\mathbf{r}, t)=-\frac{1}{A} \sum_{\mathbf{q}} g_{\mathbf{q}}^{S}(z) e^{\mathbf{i q} \cdot \mathbf{r}_{\|}} \chi_{\mathbf{q}}^{S}(t) \Theta(-z),
$$

the operators $\chi_{\mathbf{q}, p}^{B}(t)$ and $\chi_{\mathbf{q}}^{S}(t)$ being given by Eqs. (56) and (57), respectively, and $g_{\mathbf{q}, p}^{B}(z)$ and $g_{\mathbf{q}}^{S}(z)$ representing bulk and surface coupling functions,

$$
\begin{aligned}
g_{\mathbf{q}, p}^{B}(z)= & \left\{\frac{2 \omega_{\mathrm{p}} /\left(\pi \omega_{\mathbf{q}, p}^{B}\right)}{\left[2\left(\omega_{\mathbf{q}, p}^{B}\right)^{2}-\omega_{\mathrm{p}}^{2}\right]^{2}-4 \beta^{2} q^{2}\left(\omega_{q, p}^{B}\right)^{2}}\right\}^{1 / 2} \\
& \times\left\{p\left[\omega_{\mathrm{p}}^{2}+2 \beta^{2}\left(p^{2}+q^{2}\right)\right] \cos p z+q \omega_{\mathrm{p}}^{2} \sin p z\right\}
\end{aligned}
$$

and

$$
g_{\mathbf{q}}^{S}(z)=\sqrt{q \gamma_{\mathbf{q}} /\left(\omega_{\mathbf{q}}^{S} \pi\right)} \omega_{\mathbf{p}} \frac{q+\gamma_{\mathbf{q}}}{\left(q+2 \gamma_{\mathbf{q}}\right)^{1 / 2}} e^{\gamma_{\mathbf{q}^{z}}} .
$$

The electron density induced by the presence of the external perturbing charge is obtained as the expectation value of the total electron density operator. Up to first order in the external perturbation and in the limit as $z_{0} \rightarrow \infty$, we find

$$
n_{1}(z)=n_{1}^{B}(z)+n_{1}^{S}(z)
$$

where

$$
n_{1}^{B}(z)=Z_{1} \frac{\omega_{\mathbf{p}}}{2 \pi \beta} \frac{e^{\omega_{\mathrm{p}} z / \beta}-e^{\omega_{\mathrm{p}} z /(\sqrt{2} \beta)} / \sqrt{2}}{z_{0}^{2}}
$$

and

$$
n_{1}^{S}(z)=Z_{1} \frac{\omega_{\mathbf{p}}}{2 \pi \beta} \frac{e^{\omega_{\mathrm{p}} z /(\sqrt{2} \beta)} / \sqrt{2}}{z_{0}^{2}} .
$$

Equation (A6) coincides with Eq. (A1), and shows that as long as the stationary charged particle is located far from the surface the total electron density $n_{1}$ induced at the surface $(z \sim 0)$ is, in the limit as $z_{0} \rightarrow \infty, \sqrt{2}$ times the electron density $n_{1}^{S}$ induced through coupling of the charged particle with the surface plasmon field.
${ }^{1}$ See, e.g., P.J. Feibelman, Prog. Surf. Sci. 12, 287 (1982); H. Raether, Surface Plasmons, Springer Tracts in Modern Physics, Vol. 111 (Springer, Berlin, 1988).

${ }^{2}$ P.M. Echenique, F. Flores, and R.H. Ritchie, in Solid State Physics: Advances in Research and Applications, edited by H. Ehrenreich and D. Turnbull (Academic, New York, 1990), Vol. 43, p. 229.

${ }^{3}$ D.H. Schneider and M.A. Briere, Phys. Scr. 53, 228 (1996).

${ }^{4}$ A. Arnau et al., Surf. Sci. Rep. 27, 113 (1997).

${ }^{5}$ D. Niemann, M. Grether, A. Spieler, N. Stolterfoht, C. Lemell, F. Aumayr, and H.P. Winter, Phys. Rev. A 56, 4774 (1997).

${ }^{6}$ D. Niemann, M. Grether, M. Rösler, and N. Stolterfoht, Phys. Rev. Lett. 80, 3328 (1998).

${ }^{7}$ M. Hattass, T. Schenkel, A.V. Hamza, A.V. Barnes, M.W. Newman, J.W. McDonald, T.R. Niedermayr, G.A. Machicoane, and D.H. Schneider, Phys. Rev. Lett. 82, 4795 (1999).

${ }^{8}$ N. Bohr, Mat. Fys. Medd. K. Dan. Vidensk. Selsk. 18 (8), 1 (1948).

${ }^{9}$ P.M. Echenique, R.M. Nieminen, and R.H. Ritchie, Solid State Commun. 37, 779 (1981); P.M. Echenique, R.M. Nieminen, A.C. Ashley, and R.H. Ritchie, Phys. Rev. A 33, 897 (1986).

${ }^{10}$ H. Esbensen and P. Sigmund, Ann. Phys. (N.Y.) 201, 152 (1990).
${ }^{11}$ J.M. Pitarke, R.H. Ritchie, and P.M. Echenique, Nucl. Instrum. Methods Phys. Res. B 24, 613 (1993); J.M. Pitarke, R.H. Ritchie, P.M. Echenique, and E. Zaremba, Europhys. Lett. 24, 613 (1993).

12 J.M. Pitarke, R.H. Ritchie, and P.M. Echenique, Phys. Rev. B 52, 13883 (1995).

${ }^{13}$ See, e. g., P.M. Echenique, F.J. García de Abajo, V.H. Ponce, and M.E. Uranga, Nucl. Instrum. Methods Phys. Res. B 96, 583 (1995).

${ }^{14}$ J.D. Jackson, Classical Electrodynamics, 2nd. ed. (Wiley, New York, 1965).

${ }^{15}$ P.M. Echenique, R.H. Ritchie, N. Barberán, and J. Inkson, Phys. Rev. B 23, 6486 (1981).

${ }^{16}$ A.A. Lucas, Phys. Rev. B 4, 2939 (1971).

${ }^{17}$ R.H. Ritchie, Phys. Lett. A 38, 189 (1972).

${ }^{18}$ M. Sunjic, G. Toulouse, and A.A. Lucas, Solid State Commun. 11, 1629 (1972).

${ }^{19}$ J.R. Manson and R.H. Ritchie, Phys. Rev. B 24, 4867 (1981).

${ }^{20}$ F.J. García de Abajo and P.M. Echenique, Phys. Rev. B 46, 2663 (1992).

${ }^{21}$ X.-Y. Zheng, R.H. Ritchie, and J.R. Manson, Phys. Rev. B 39, 13510 (1989). 
${ }^{22}$ A. Bergara, J.M. Pitarke, and F.J. García de Abajo, Nucl. Instrum. Methods Phys. Res. B 135, 97 (1998).

${ }^{23}$ R.H. Ritchie and R.E. Wilems, Phys. Rev. 178, 372 (1969).

${ }^{24}$ A.G. Eguiluz, Phys. Rev. Lett. 51, 1907 (1983).

${ }^{25}$ R.H. Ritchie, Phys. Rev. 106, 874 (1957); Surf. Sci. 34, 1 (1973).

${ }^{26}$ G. Barton, Rep. Prog. Phys. 42, 963 (1979).

${ }^{27}$ A. Bergara, J.M. Pitarke, and R.H. Ritchie, Nucl. Instrum. Methods Phys. Res. B 115, 70 (1996).

${ }^{28}$ L. Hedin and S. Lundqvist, in Solid State Physics: Advances in Research and Applications, edited by F. Seitz, D. Turnbull, and H. Ehrenreich (Academic, New York, 1969), Vol. 23, p. 1.

${ }^{29}$ J.M. Pitarke, A. Bergara, and R.H. Ritchie, Nucl. Instrum. Methods Phys. Res. B 99, 87 (1995); A. Bergara, I. Campillo, J.M. Pitarke, and P.M. Echenique, Phys. Rev. B 56, 15654 (1997).

${ }^{30} \mathrm{~S}$. Lundqvist, in Theory of the Inhomogeneous Electron Gas, ed- ited by S. Lundqvist and N.H. March (Plenum, New York, 1983), p. 149.

${ }^{31}$ S.C. Ying, Nuovo Cimento Soc. Ital. Fis., B 23, 270 (1974).

${ }^{32}$ R.H. Ritchie, Prog. Theor. Phys. 29, 607 (1963).

${ }^{33}$ P. Halevi, Phys. Rev. B 51, 7497 (1995).

${ }^{34}$ R.H. Ritchie and A.L. Marusak, Surf. Sci. 4, 234 (1966).

${ }^{35}$ A. Griffin and J. Harris, Can. J. Phys. 54, 1396 (1976).

${ }^{36}$ A.G. Eguiluz, Solid State Commun. 33, 21 (1980); Phys. Rev. B 23, 1542 (1981)

${ }^{37}$ A.L. Fetter and J.D. Walecka, Quantum Theory of Many-Particle Systems (McGraw-Hill, New York, 1971).

${ }^{38}$ A. Bergara, J.M. Pitarke, and R.H. Ritchie, Phys. Lett. A 256, 405 (1999).

${ }^{39}$ The one-electron radius $r_{s}$ is defined by the relation $n_{0}$ $=3 /\left(4 \pi r_{s}^{3}\right)$. 